\title{
Use of transesophageal echocardiography for transcatheter valve- in-valve implantation for patients with prior bioprosthetic surgical aortic, mitral, tricuspid, and pulmonic valves
}

\author{
Ankit A. Bhargava ${ }^{1}$, Nikoloz Shekiladze ${ }^{1}$,Joe Xie ${ }^{1}$, Annapoorna Kini ${ }^{2}$, Patrick T. Gleason ${ }^{1 \#,}$ \\ Stamatios Lerakis ${ }^{2 \#}$ \\ ${ }^{1}$ Division of Cardiology, Emory University School of Medicine, Atlanta, GA, USA; ${ }^{2}$ Division of Cardiology, Icahn School of Medicine at Mount \\ Sinai, New York, NY, USA \\ \#These authors contributed equally to this work as senior authors. \\ Correspondence to: Patrick T. Gleason, MD. Division of Cardiology, Emory University School of Medicine, 550 Peachtree Street, Atlanta, GA 30308, \\ USA. Email: Patrick.t.gleason@emory.edu; Stamatios Lerakis, MD. Division of Cardiology, Icahn School of Medicine at Mount Sinai, 1468 Madison \\ Avenue, New York, NY 10029, USA. Email: Stamatios.lerakis@mountsinai.org.
}

\begin{abstract}
Valve-in-valve $(\mathrm{ViV})$ transcatheter procedures are the preferred option for redo valve replacement in patients who otherwise would be high risk for surgery. Transesophageal echocardiography (TEE) is an integral imaging modality for both peri-procedural and intra-procedural guidance during transcatheter ViV replacement. When intentional leaflet laceration is needed, such as with the BASILICA (Bioprosthetic or native Aortic Scallop Intentional Laceration to prevent Iatrogenic Coronary Artery obstructions during TAVR) or LAMPOON (Laceration of the Anterior Mitral valve leaflet to Prevent left ventricular Outlet ObstructioN) procedures, TEE is critical to proper guidewire positioning and achieving a successful laceration. In this paper we detail the role of TEE in $\mathrm{ViV}$ transcatheter valve replacement in patients with prior surgical aortic, mitral, tricuspid, and pulmonic valves.
\end{abstract}

Keywords: Valve-in-valve (ViV); transcatheter aortic valve replacement (TAVR); transcatheter mitral valve replacement (TMVR); BASILICA; LAMPOON

Submitted Jul 27, 2021. Accepted for publication Aug 18, 2021.

doi: 10.21037/acs-2021-tviv-27

View this article at: https://dx.doi.org/10.21037/acs-2021-tviv-27

\section{Introduction}

The last decade has seen an increase in patient preference for bioprosthetic valves over mechanical valves in those undergoing valve replacement (1). By nature of their biologic material, bioprosthetic valves are more prone to degeneration and do not have the same durability as mechanical valves. The incidence of structural valve degeneration of bioprosthetic valves increases substantially 7 to 8 years after initial valve replacement (2). Consequently, there are increasingly more patients needing repeat valve intervention. However, the risks of a redo valve operation may be prohibitive due to advanced age and other comorbidities $(3,4)$. The valve-in-valve $(\mathrm{ViV})$ procedure using a transcatheter valve and delivery system offers a minimally invasive approach towards repeat valve replacement that has proven to be an attractive option for this patient population.

Transesophageal echocardiography (TEE) is often needed during a ViV intervention and has different applications depending on the valve that needs to be replaced. TEE provides valuable real-time imaging for accurate delivery of various valves. At the same time, it identifies potential complications that can then either be prevented or dealt with promptly. In the following sections, we detail our approach to TEE image acquisition in ViV interventions for aortic, mitral, tricuspid, and pulmonic positions. 


\section{Protocol for pre-procedural TEE image acquisition}

Although the sequences for TEE image acquisition may differ by institution, it is important to have a protocolized approach for pre-procedural TEE image acquisition. Expertise in multiplane imaging and three-dimensional (3D) imaging is also essential for guidance during $\mathrm{ViV}$ procedures.

Multiplane imaging (also known as X-plane imaging) allows for the simultaneous display of orthogonal plane images. X-plane is heavily utilized both periprocedurally and intra-procedurally. 3D imaging allows for comprehensive assessment of valve anatomy and pathology. Depending on the target valve, this modality may be essential to intra-procedural guidance as well.

The pre-procedural TEE should aim to accomplish the following:

(I) To confirm the pathology of the prosthetic valve;

(II) To obtain baseline assessments of various cardiac structures, including left ventricle (LV) and right ventricle (RV) size and function, presence of pericardial effusion, aortic root anatomy, and presence of left atrial appendage (LAA) thrombus;

(III) To locate the ideal patient-specific TEE imaging windows for intra-procedural guidance during valve intervention;

(IV) To serve as a comparison to post-intervention images.

Figure 1 outlines our suggested pre-procedural protocol. Depending on the valve intervention, certain steps are expanded upon and will be discussed in their respective sections.

\section{Role of TEE for ViV procedure in prior surgical aortic valve replacement (SAVR)}

In patients with a prior SAVR, pre-procedural planning with multidetector computed tomography (MDCT) identifies patients in whom $\mathrm{ViV}$ transcatheter aortic valve replacement (TAVR) may be performed safely with low risk of complication or pose a risk of coronary obstruction (Figure 2). MDCT can give accurate measurements of coronary height from the aortic valve annulus, model a virtual valve to determine the valve to coronary (VTC) and valve to sinotubular junction (VTSTJ) distances, and identify the type of bioprosthetic valve present in patients who lack this data. Major risk factors that predispose to coronary obstruction from transcatheter valve placement include low coronary height, prosthetic leaflets that extend higher than the coronary ostia with a VTC distance $<4 \mathrm{~mm}$, and sealing of the sinotubular junction by visual assessment of the virtual valve (5). If MDCT determines that the patient is at low risk for coronary obstruction with $\mathrm{ViV}$ TAVR, then intraprocedural TEE is often not needed. In these cases, the procedures are guided by fluoroscopy given that the radiographic markers are usually readily seen, and transthoracic echocardiography can be used to assess the valve before and after the procedure.

In patients who are at risk of coronary obstruction, the BASILICA (Bioprosthetic or native Aortic Scallop Intentional Laceration to prevent Iatrogenic Coronary Artery obstructions during TAVR) procedure is a potential strategy for mitigating this risk. TEE is essential in aortic $\mathrm{ViV}$ procedures where BASILICA is the planned method for reducing the risk of coronary obstruction prior to TAVR implantation.

\section{BASILICA and TEE guidance}

Coronary obstruction is a rare, but life-threatening complication after TAVR. In patients undergoing ViV TAVR, the mortality rate after coronary obstruction is $48.6 \%$ (6). The risk of coronary obstruction is higher in $\mathrm{ViV}$ procedures as compared to the risk of obstruction in native aortic valves $(2.3 \%$ vs. $0.7 \%)$ (6). Obstruction is nearly always due to displacement of the valve leaflets towards the coronary ostia (6).

The BASILICA procedure utilizes two guiding catheters to form a loop of electrified guidewire positioned at the base of the target leaflet and left ventricular outflow tract (LVOT) to then lacerate the leaflet. As a result, the leaflet is splayed open and does not obstruct coronary flow (7).

The pre-procedural TEE follows the described steps in Figure 1, with additional emphasis on the assessment of the bioprosthetic aortic valve, the coronary ostial positions, the baseline LV ejection fraction, and the presence of any wall motion abnormalities.

When assessing the bioprosthetic aortic valve, the first step is to confirm the valve pathology. This includes identification of leaflet degeneration and/or calcification, the baseline severity of stenosis and/or insufficiency, the presence of paravalvular leak, the presence of thrombus or pannus, and the presence of a mass or vegetation. In patients with prosthetic valve regurgitation, the target coronary leaflet should be carefully inspected for any tears 


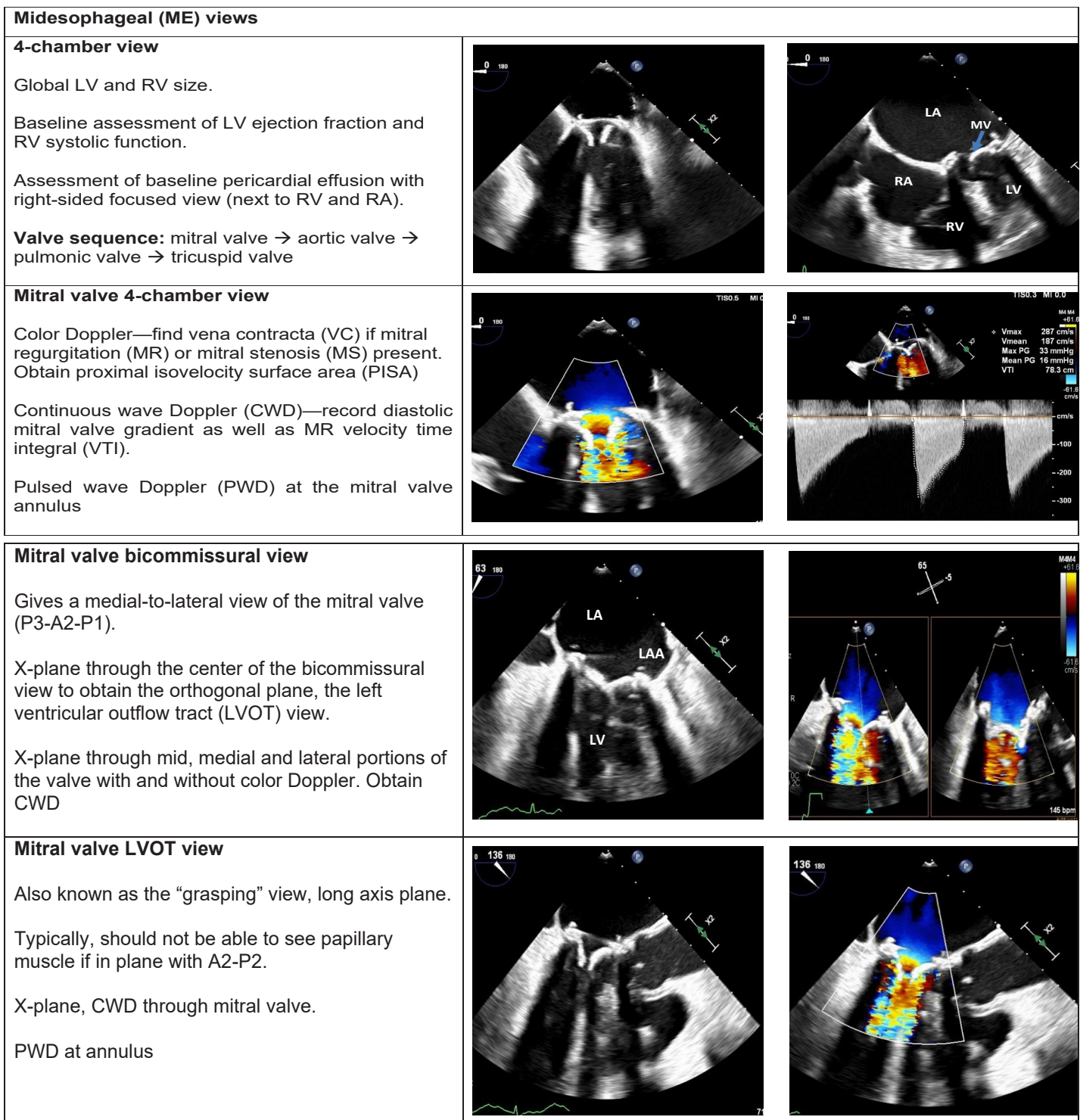




3D surgeon's view
Obtained while in the LVOT view, make sure to
include portion of aortic valve.
The smaller the pyramid of data, the higher the
frame rate.
Once in 3D, orient the aortic valve to the 12 o'clock
position. This will put the mitral valve in an anterior-
posterior axis. The left portion of the valve is
lateral, the right portion of the valve is medial.
Obtain 3D color images $\rightarrow$ goal frame rate is $\geq 10$
Hz.
Record simultaneous atrial and ventricular views in
3D
Aortic valve long axis view
Obtained from the LVOT view, requires anteflexion
and clocking of the probe.
Record with and without color Doppler. Clockwise
and counterclockwise turn through the valve.
annulus to assess anatomy/pathology around
aortic valve
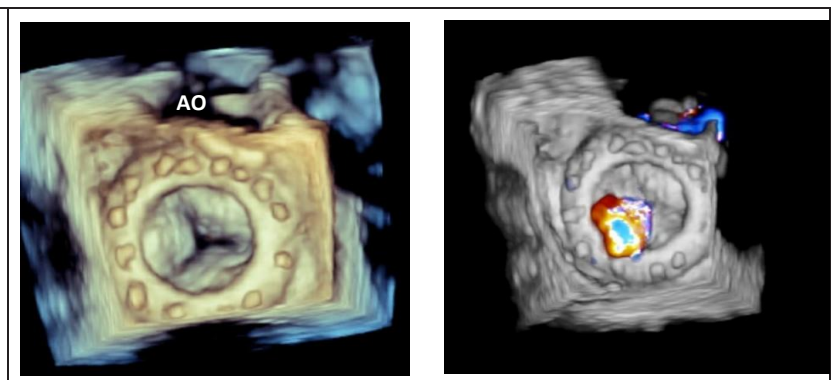

Aortic valve short axis
Subtract 90 degrees from long axis view while
keeping aorta in the center of the image to get the
basal short axis.
Record with and without color Doppler
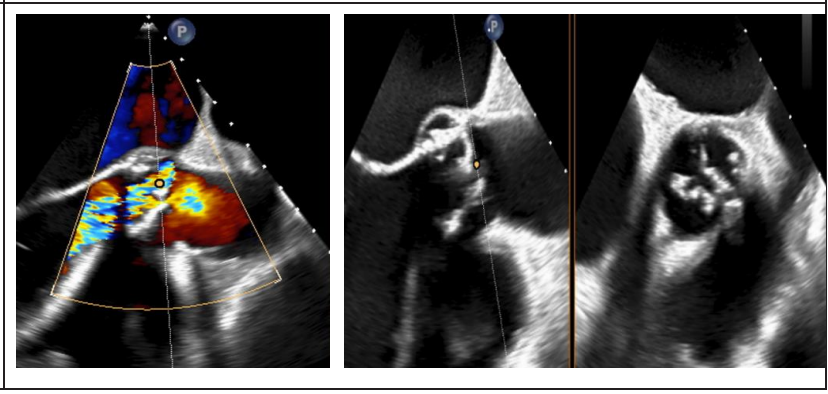

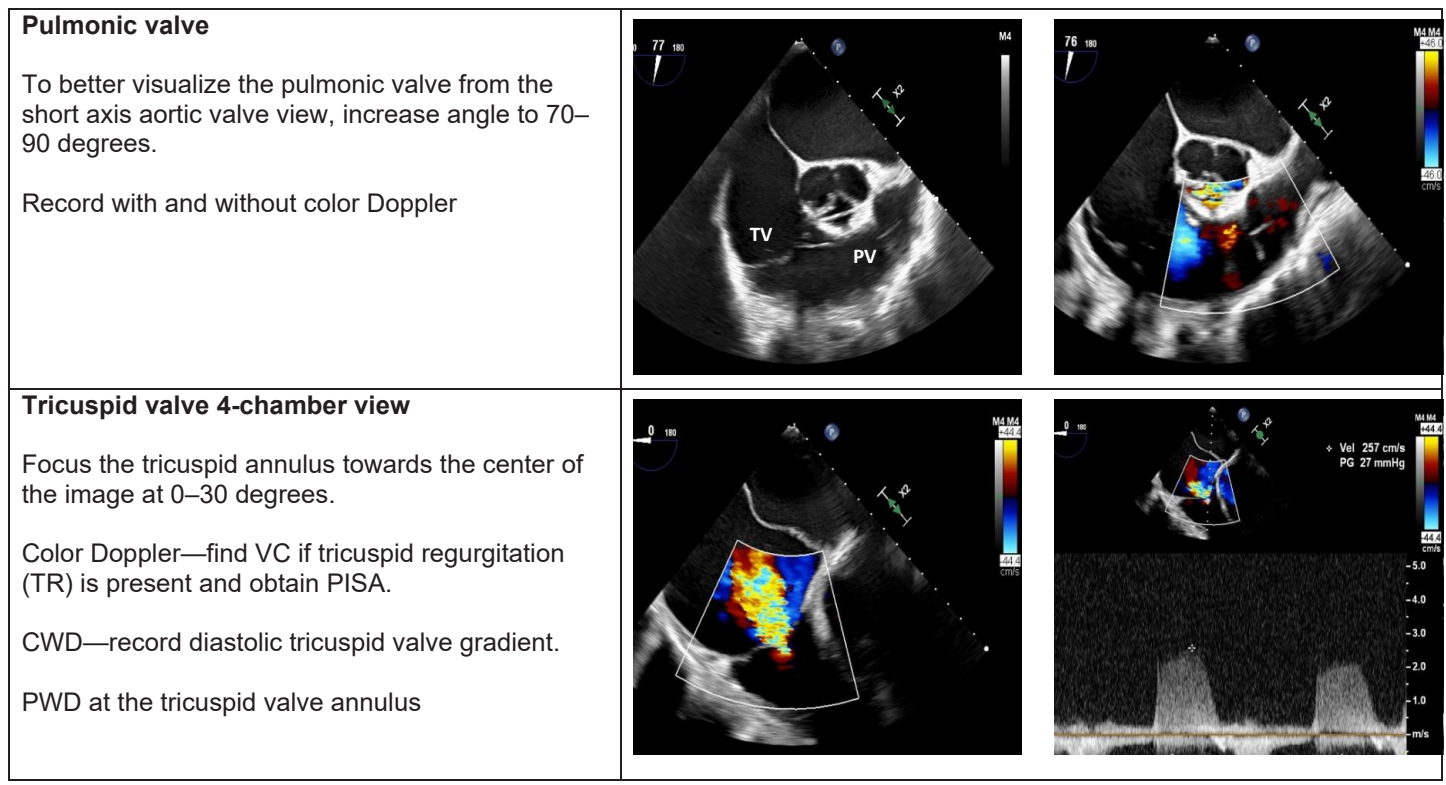




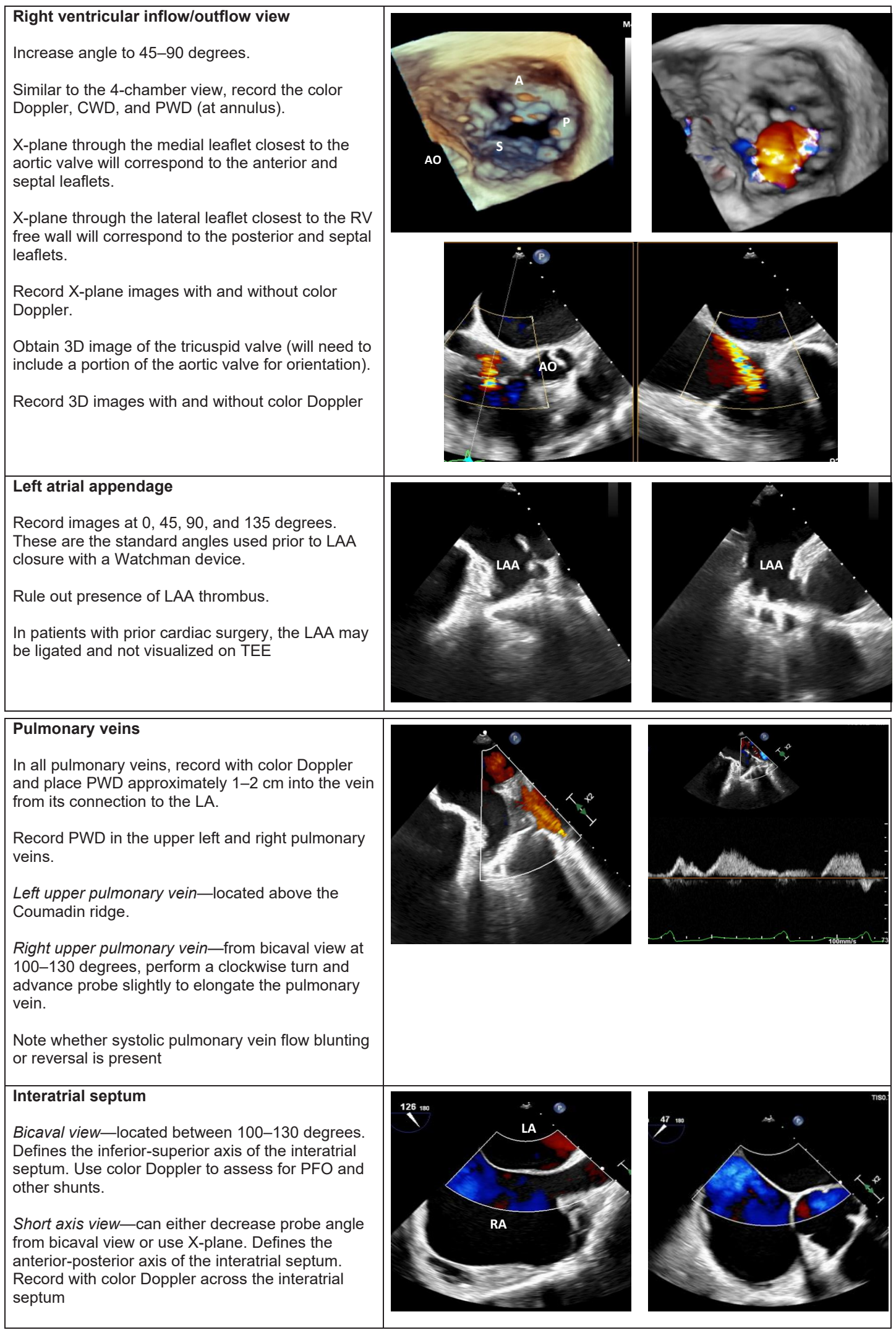




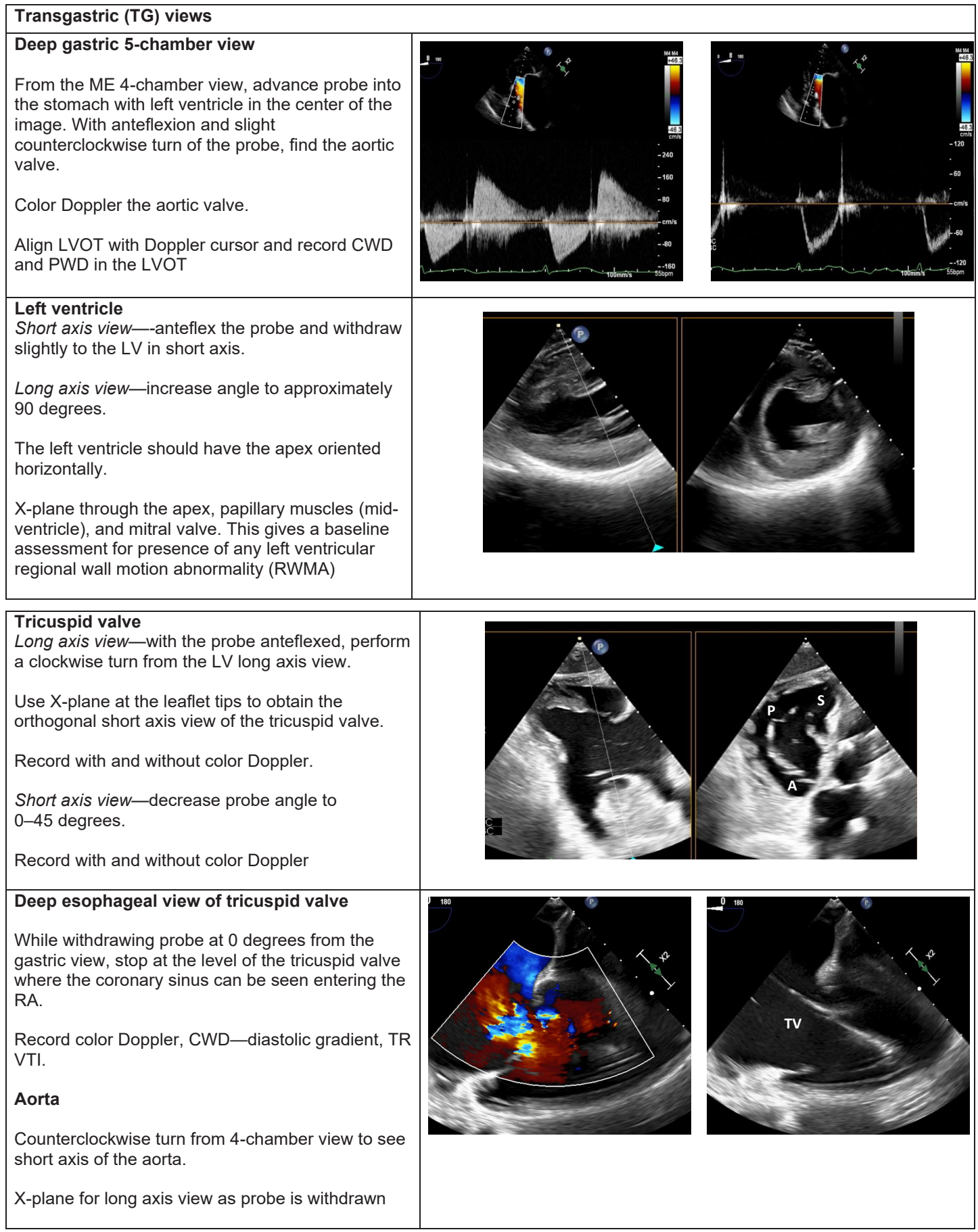

Figure 1 Suggested pre-procedural TEE image acquisition sequence. ME, midesophageal; LA, left atrium; LV, left ventricle; RV, right ventricle; RA, right atrium; VC, vena contracta; MR, mitral regurgitation; MS, mitral stenosis; PISA, proximal isovelocity surface area; CWD, continuous wave Doppler; VTI, velocity time integral; PWD, pulsed wave Doppler; LAA, left atrial appendage; LVOT, left ventricular outflow tract; 3D, three-dimensional; AO, aorta; TR, tricuspid regurgitation; TEE, transesophageal echocardiography; TG, transgastric; RWMA, regional wall motion abnormality. 

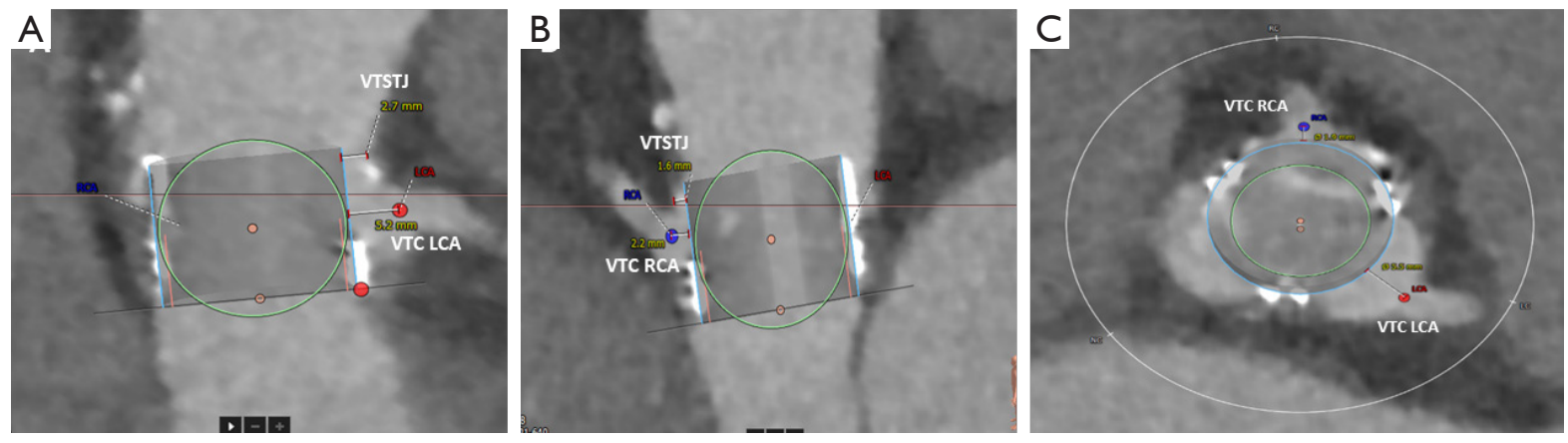

Figure 2 MDCT pre-procedural planning for transcatheter ViV TAVR with measurements of the VTC and VTSTJ. Depiction of virtual valve in an aortic prosthetic valve with measurements of the VTC and VTSTJ in the long axis (A,B) and short axis (C). The right coronary artery is at risk of coronary obstruction due to its low coronary height, VTC distance $<4 \mathrm{~mm}$, and the close proximity of the virtual valve to the sinotubular junction. MDCT, multidetector computed tomography; ViV, valve-in-valve; TAVR, transcatheter aortic valve replacement; VTC, valve to coronary; VTSTJ, valve to sinotubular junction.

or lacerations, as this could prohibit proper alignment and execution of the BASILICA (8).

After valve assessment, the probe may need to be withdrawn slightly to assess the position of the coronary ostia. The coronary ostia can be centrally or eccentrically positioned within the coronary cusp. The Nyquist limit is decreased $(25-30 \mathrm{~cm} / \mathrm{sec})$ to visualize coronary flow. The right coronary artery may be challenging to visualize due to acoustic shadowing from the aortic valve prosthesis. Ostial eccentricity tends to be more common for the right coronary cusp and can be more easily identified on preprocedure MDCT (9).

Lastly, accurate assessment of the left ventricular ejection fraction and wall motion should be recorded. After deployment of the TAVR valve within the existing prosthesis, loss of coronary color flow or a new regional wall motion abnormality (RWMA) may indicate obstruction by a prosthetic valve leaflet.

At the time of the procedure, the TEE is key for the following steps (8) (Figure 3):

(I) Positioning of the snare system into the LV;

(II) Positioning of the traversal wires;

(III) Snare wire and formation of the "flying V";

(IV) Laceration of the prosthetic leaflet(s);

(V) Post-TAVR implantation assessment.

\section{Positioning of the snare in the $\mathbf{L V}$}

During the snare positioning step, a multipurpose (MP) guide catheter with a stabilizing anchor wire and a singleloop snare are advanced across the aortic valve. The anchor wire is placed at the LV apex while the snare loop wire is positioned in the LVOT. To visualize the wire and snare, begin with the midesophageal (ME) long axis aortic valve view and use the $\mathrm{X}$-plane tool to obtain the orthogonal short axis view of the aortic valve. For the wire and catheter to be in a single plane of imaging in the long axis view, slight clockwise or counterclockwise turns of the TEE probe, along with angle adjustment, may be needed. The $\mathrm{X}$-plane should cut through the level of the aortic valve to show the position of the MP catheter inside the valve. The orthogonal short axis view should be oriented in the standard 2D short axis aortic valve view. The trajectory of the anchoring wire towards the $L V$ apex can be seen in the long axis LVOT view. Though challenging, the snare wire may be seen below the aortic valve annulus.

\section{Positioning of the traversal system}

To position the traversal system, a curved traversal catheter, which contains an electrifiable guidewire, is placed in the target coronary cusp. The electrifiable guidewire is advanced towards the base of the target leaflet and in front of the coronary ostium. An electric current is applied to the distal end of the guidewire, allowing it to puncture through the leaflet and advance into the LVOT.

Starting with the long axis aortic valve view with $\mathrm{X}$-plane, the traversal catheter tip is guided towards the base of the leaflet at the position in front of, and proximal to, the coronary ostium. Using X-plane at the level of the catheter tip is crucial for proper positioning, especially in eccentrically positioned coronary ostia. The long axis view 

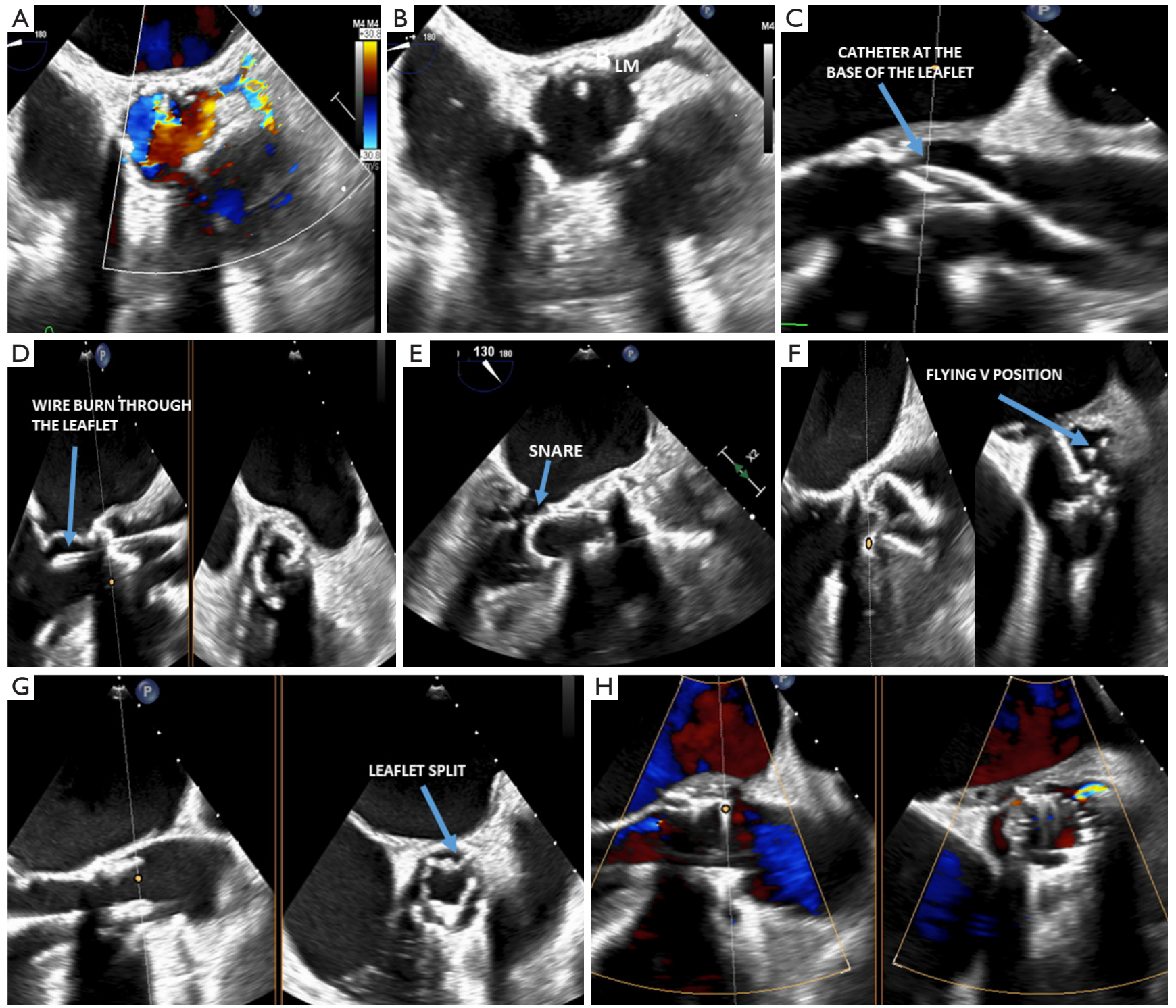

Figure 3 TEE images for transcatheter ViV TAVR with BASILICA. (A,B) Pre-procedural TEE with demonstration of color Doppler flow in the left coronary artery. (C) TEE-guided catheter positioning at the base of the aortic leaflet in the long axis aortic valve view. (D) Visualization of the traversal wire in the LVOT after burning through the base of the leaflet with orthogonal short axis view below the aortic valve annulus. (E) Visualization of the snare wire in the LVOT. (F) Formation of the flying V with simultaneous long and short axis views confirming the appropriate position of the wire in front of the coronary ostium prior to laceration. (G) Post-laceration of the prosthetic aortic valve leaflet in long and short axis views. (H) Post-implantation of TAVR valve with color flow Doppler seen after successful ViV deployment. TEE, transesophageal echocardiography; ViV, valve-in-valve; TAVR, transcatheter aortic valve replacement; BASILICA, Bioprosthetic or native Aortic Scallop Intentional Laceration to prevent Iatrogenic Coronary Artery obstructions during TAVR; LVOT, left ventricular outflow tract.

is used to confirm the trajectory of the traversal guidewire into the LVOT, ensuring that no adjacent structures—such as the aortic root, mitral valve, atria, or interventricular septum—are at risk of injury. Upon applying the electric current, heat-induced evaporation causes bubbles to fill the aortic root and obscures the view of the guidewire as it traverses the leaflet. While the traversal itself is not well seen, the guidewire catheter tip should ultimately be seen 
in the LVOT. The anterior mitral valve leaflet and the basal anteroseptum should be inspected to confirm that they have not been inadvertently traversed by the guidewire when crossing the left and right coronary leaflets, respectively.

\section{Snare wire and formation of the flying $\mathrm{V}$}

After the guidewire traverses the prosthetic leaflet, it is snared in the LVOT and retrieved through the aortic valve in the MP catheter, thereby straddling the aortic scallop and forming the "flying V." The flying $\mathrm{V}$ is positioned at the expected point of laceration.

On TEE, the actual snaring of the traversal wire is difficult to see due to reverberation artifact from multiple wires in a confined space. During this step, the mitral valve should also be assessed in the ME long axis view for regurgitation in case of accidental chordal entrapment. This can occur if the traversal wire is snared too low in the LV or LVOT. After successful snaring, the flying $\mathrm{V}$ is positioned radially towards the coronary ostium, typically in the center of the cusp, to properly align the guidewire laceration of the leaflet. The traversal catheter portion of the $\mathrm{V}$ is oriented along the aortic side of the leaflet, while the MP catheter portion of the $\mathrm{V}$ is oriented along the ventricular side. The ME short axis aortic valve view confirms that the radial position of the flying $\mathrm{V}$ is in line with the coronary ostium and away from the valve commissures. An asymmetric laceration due to poor positioning of the flying $\mathrm{V}$ can leave residual prosthetic leaflet material for coronary obstruction $(7,8)$.

\section{Laceration of the prosthetic leaflet}

When ready for laceration, the MP and traversal catheters are pulled upwards while electricity is applied to the flying $\mathrm{V}$ guidewire, splaying open the leaflet prosthesis. Similar to the leaflet traversal step, bubbles fill the aortic root on TEE and obscure the visualization of leaflet laceration. A pigtail catheter is typically placed in the $\mathrm{LV}$ prior to leaflet laceration to facilitate TAVR deployment. In the ME $\mathrm{LV}$ axis view, check that the pigtail catheter has not been dislodged from the LV apex. Color Doppler of the aortic valve should reveal new or worsened aortic insufficiency secondary to leaflet laceration. The short axis aortic valve view confirms that proper leaflet laceration has occurred. This is evidenced by a radial laceration towards the target coronary ostium extending from the tip to the base of the leaflet. If an asymmetric laceration is present, the coronary ostium may still be at risk for obstruction. Preventive measures, such as a preemptive coronary stent placement, may be needed.

\section{Post-TAVR implantation}

Once the TAVR valve is deployed, the short axis aortic valve view is attained to examine coronary perfusion with color Doppler at a reduced Nyquist limit of $25-30 \mathrm{~cm} / \mathrm{sec}$. Occasionally, partial leaflet prolapse can be seen going into the coronary artery. The LV and RV function is reassessed and compared to baseline images. RWMA are assessed from the ME 4-chamber and transgastric views with $\mathrm{X}$-plane through the apex, papillary muscles, and basal LV. The TAVR valve is assessed for new baseline gradients, valvular insufficiency, and paravalvular leak. The ascending and descending thoracic aorta are also inspected for any evidence of dissection or hematoma formation. Other complications secondary to BASILICA, such as the presence of a new or enlarged pericardial effusion, new intracardiac shunts, and new or worsened mitral regurgitation (MR), should be excluded by TEE.

\section{Role of TEE for ViV procedure in prior surgical mitral valve replacement (SMVR)}

\section{Pre-procedure risk assessment}

In patients with a SMVR, pre-procedural planning with MDCT identifies patients in whom ViV transcatheter mitral valve replacement (TMVR) may be safely performed with low risk of complication or pose a risk for LVOT obstruction (LVOTO). MDCT is used to model a virtual balloon expandable valve in the mitral position. With this virtual valve in place, a "neo-LVOT" area can be measured $(10,11)$ (Figure 4). Patients at risk of LVOTO may be candidates for LAMPOON.

When a transcatheter valve is implanted in the surgical mitral valve, the surgical prosthetic leaflet is displaced anteriorly within the stent frame. While LVOTO is still possible, the rate of this occurrence is rare. The reported incidence of ViV LVOTO is $0.7-2.2 \%(12,13)$.

The major predictor of risk for LVOTO after TMVR is a decreased neo-LVOT area as measured by MDCT. This measurement is taken during mid to end systole. The typical cut-off range for high risk of LVOTO is a neo-LVOT area $\leq 1.7$ to $2.0 \mathrm{~cm}^{2}(10,14,15)$. The neo-LVOT area is affected by the anatomic interplay of the aortic valve, mitral valve, 

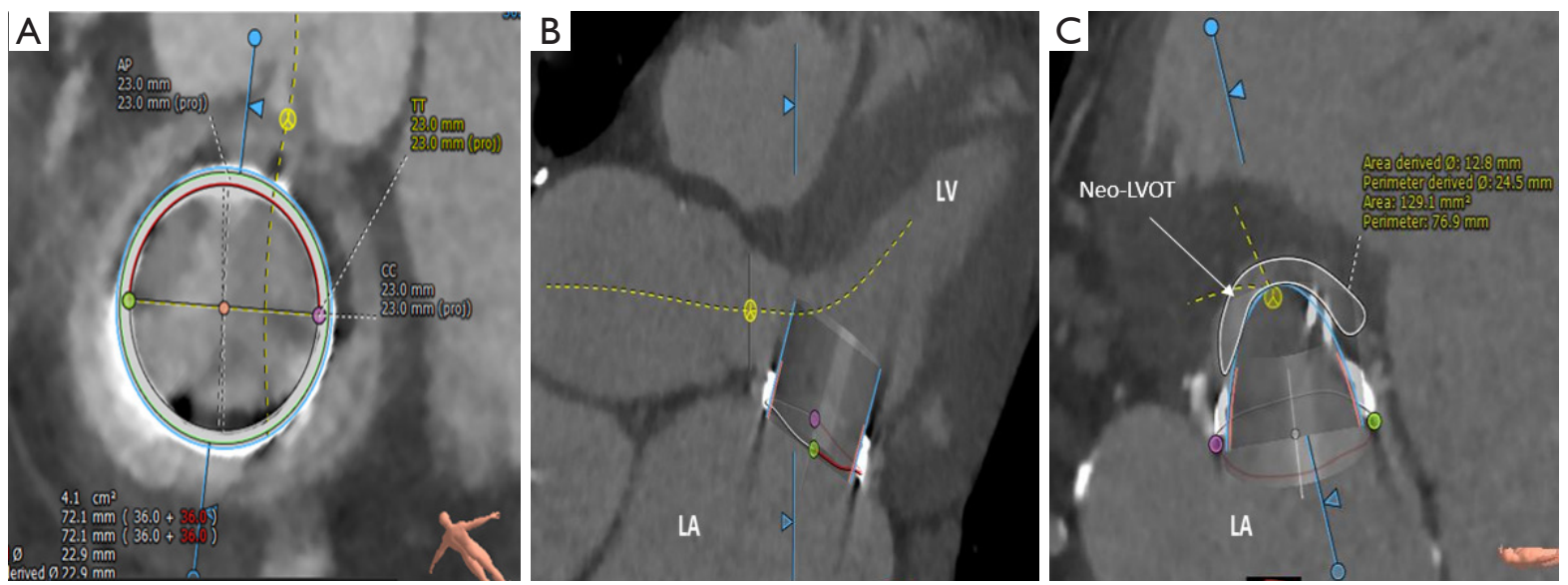

Figure 4 MDCT pre-procedural planning for ViV TMVR. (A) Depiction of virtual ViV in the prosthetic mitral valve from the short axis, (B) long axis views along the neo-LVOT track, (C) and the measured neo-LVOT area. LA, left atrium; LV, left ventricle; MDCT, multidetector computed tomography; ViV, valve-in-valve; TMVR, transcatheter mitral valve replacement; neo-LVOT, neo-left ventricular outflow tract.

and LV. Factors that predispose to a small neo-LVOT area include type and size of mitral prosthesis, a narrow aortomitral angle, a small native LVOT size, a small LV size, and LV hypertrophy.

\section{TEE and ViV TMVR without LAMPOON}

Regardless of the need for LAMPOON, TEE is crucial for procedural guidance during ViV TMVR. The transseptal puncture method is the typical approach for TMVR. Baseline images are acquired as described in Figure 1, with additional emphasis on evaluation of the mitral valve prosthesis, the interatrial septum, and measurement of the LVOT gradient $(11,16)$.

When assessing the bioprosthetic mitral valve, the first step is to confirm the valve pathology. The valve prosthesis is inspected for leaflet degeneration and/or calcification, baseline degree of stenosis and/or insufficiency, presence of paravalvular leak, presence of thrombus or pannus, degree of mitral annular calcification, and presence of a mass or vegetation. 3DTEE imaging of the mitral valve prosthesis is particularly helpful in elucidating valve pathology. The anatomical relationship of the surgical mitral bioprosthetic valve to the LVOT should also be noted.

The trans-septal puncture is a critical step to TMVR. The interatrial septum is therefore given special attention, particularly in finding the ideal TEE windows to achieve the trans-septal puncture. Potential anatomic challenges to trans-septal puncture include presence a patent foramen ovale, atrial septal defect, interatrial septal aneurysm, septal closure device, or lipomatous hypertrophy of the interatrial septum.

Measurement of the LVOT gradient is best achieved using continuous wave Doppler (CWD) from the deep gastric view. Clinically significant LVOT obstruction is defined as a peak intracavitary gradient $>30 \mathrm{mmHg}$ (17).

\section{TEE and trans-septal access}

To position the catheter for trans-septal puncture, the bicaval view is used to define the inferior-superior axis in conjunction with X-plane for the orthogonal anteriorposterior axis of the interatrial septum. The preferred position for trans-septal puncture is mid-septum in the bicaval view and posterior in the anterior-posterior view. Moving the catheter posteriorly gives the operator more height above the mitral valve annulus. Prior to puncture, the interatrial septum is tented by the guidewire. Using the 4-chamber view, the height of the puncture is measured from the mitral valve annulus. A height of $3.0-4.0 \mathrm{~cm}$ above the mitral valve annulus is acceptable for puncture. In cases with difficult anatomy, an extreme angle from 160-180 degrees may have a better visualization of this height. Once positioned, the moment of puncture is captured, and the 

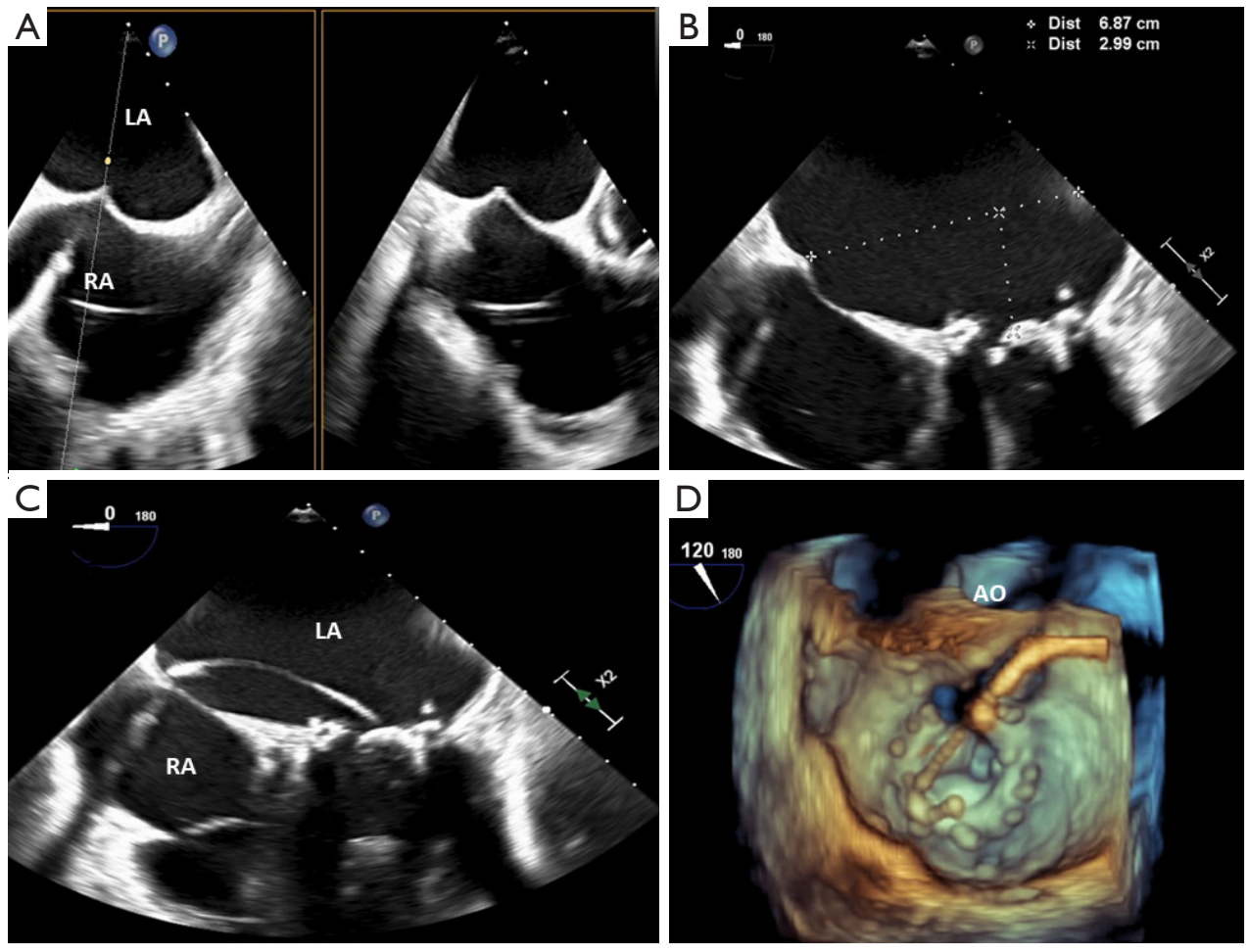

Figure 5 TEE guidance for trans-septal puncture. (A) X-plane imaging demonstrating simultaneous bicaval and anterior-posterior views with tenting of the interatrial septum by wire. (B) The height of the anticipated site of puncture is measured from the mitral valve annulus using the 4-chamber view. (C) After trans-septal puncture, the wire is seen in the LA. (D) Its position in the LA can also be seen using 3D TEE imaging. TEE, transesophageal echocardiography; 3D, three-dimensional; LA, left atrium; AO, aorta.

wire is followed into the left atrium (LA) to ensure no injury to any structures (Figure 5).

\section{TMVR valve positioning and deployment}

TEE is used in conjunction with fluoroscopy to position the transcatheter valve within the existing bioprosthesis. The ME bicommissural view is used for the medial to lateral positioning of the valve. The LVOT view is used to assess the coaxial alignment of the device to the annulus and its alignment to the surgical mitral valve prosthesis in the anterior-posterior axis (16) (Figure 6).

After deployment of the transcatheter valve into the mitral valve prosthesis, TEE is used to assess device positioning, valve function, baseline transvalvular gradients, valvular regurgitation, paravalvular leak, new baseline LVOT gradient, and LV/RV size and function. Towards the end of the procedure, the iatrogenic interatrial septal shunt is evaluated for size and directionality of shunt. While not routinely closed, the presence of a significant right-to- left shunt or pulmonary hypertension with RV failure may necessitate deployment of a percutaneous closure device into the shunt (18) (Figure 7).

TEE is used to assess for the following complications for ViV TMVR after valve deployment into the surgical prosthesis:

(I) New or enlarged pericardial effusion;

(II) New RWMA secondary to compression of the left circumflex artery after valve deployment;

(III) LVOTO;

(IV) Interatrial septal rupture.

\section{TEE considerations for ViV TMVR with LAMPOON}

ViV TMVR with LAMPOON follows the same transseptal approach as described above. After trans-septal puncture, a guide sheath is advanced across the interatrial septum and into the LA. Through this guide sheath, a catheter is directed across the mitral valve. In patients with degenerated bioprosthetic valves with severe stenosis, 

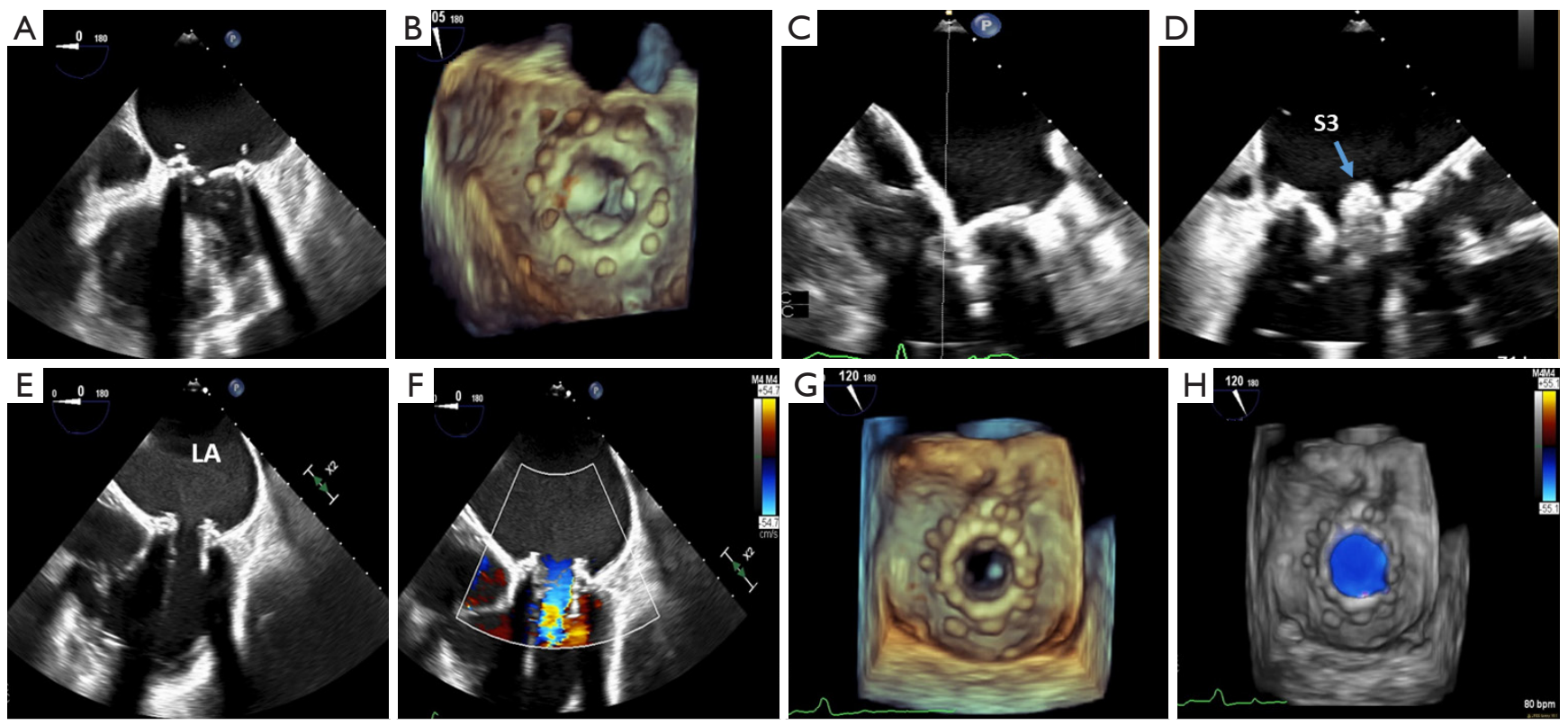

Figure 6 TEE guidance of transcatheter valve into the surgical mitral valve prosthesis. Baseline 2D (A) and 3D (B) TEE images of a severely degenerated surgical mitral valve prosthesis. After trans-septal puncture, the transcatheter valve is positioned into surgical mitral valve prosthesis using the bicommissural (C) and LVOT long axis views (D) for medial-lateral and anterior-posterior alignment, respectively. 2D TEE images of post-deployment ViV TMVR without color (E) and with color Doppler (F). 3D TEE images of ViV TMVR without color (G) and with color Doppler (H). TEE, transesophageal echocardiography; 2D, two-dimensional; 3D, three-dimensional; LVOT, left ventricular outflow tract; ViV, valve-in-valve; TMVR, transcatheter mitral valve replacement.
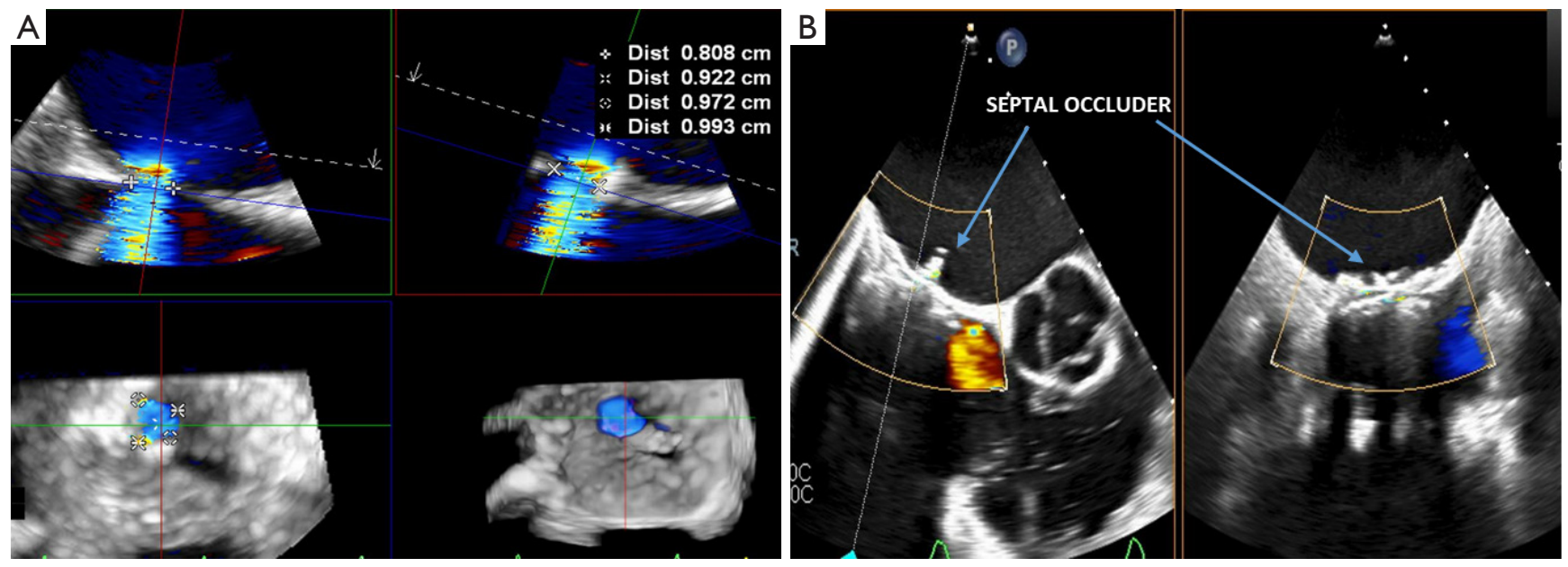

Figure 7 TEE assessment of the interatrial septum post-deployment of transcatheter valve and removal of trans-septal delivery system. (A) The size and directionality of the iatrogenic interatrial shunt is assessed with multiplane TEE imaging and color Doppler. (B) A septal occluder device is deployed across the shunt with minimal color flow seen around or through the device. TEE, transesophageal echocardiography. 

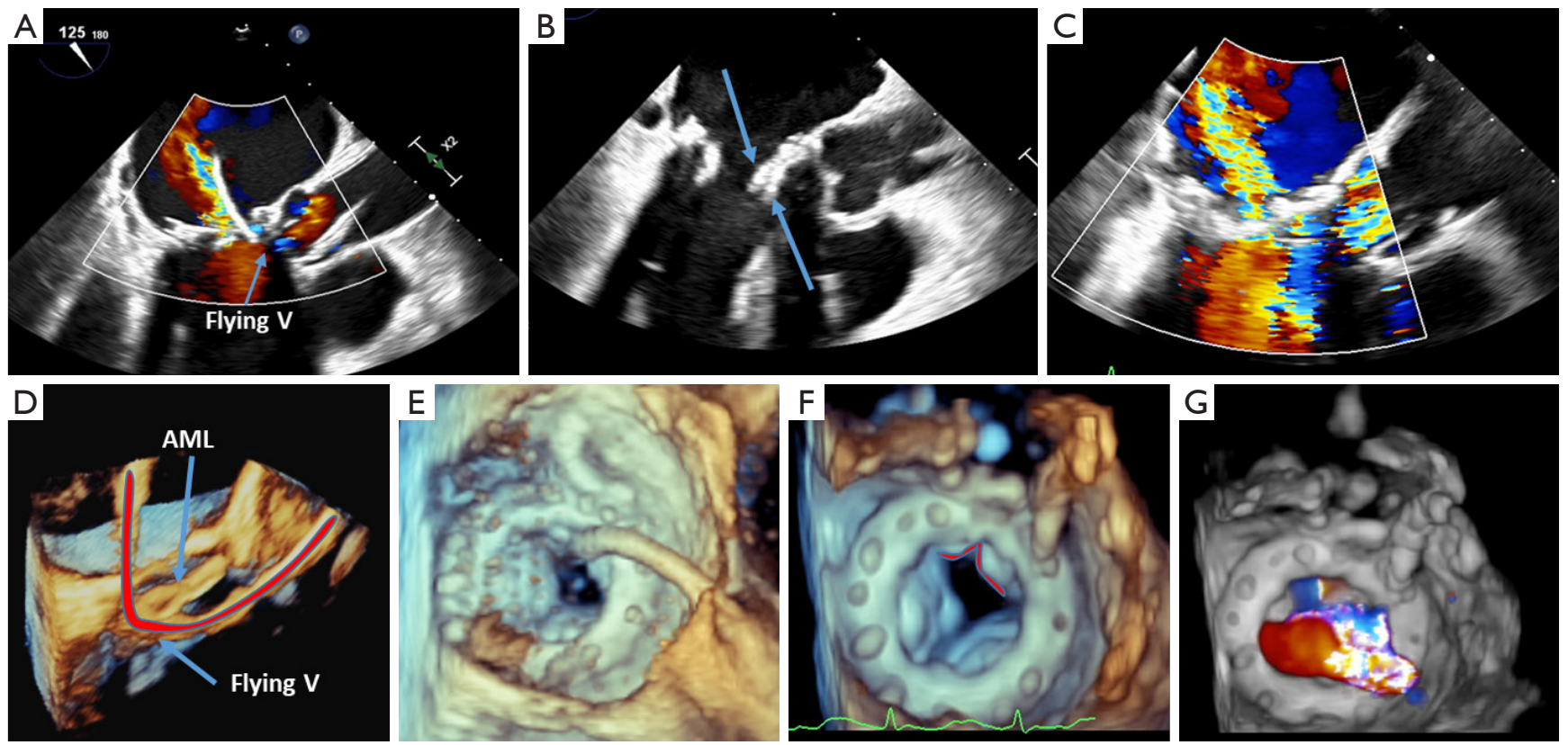

Figure 8 TEE guidance of tip-to-base LAMPOON. (A) The trans-septal wire is seen connecting the left atrial and aortic catheters with tension on the anterior mitral valve prosthetic leaflet. After laceration, (B) this prosthetic leaflet should be splayed open (C) with worsening of MR by color Doppler. (D) 3D TEE images with formation of the flying $\mathrm{V}$ in the LVOT long axis view (E) and central positioning on the prosthetic leaflet. (F) 3D depiction of leaflet laceration (G) and worsened MR. TEE, transesophageal echocardiography; LAMPOON, Laceration of the Anterior Mitral valve leaflet to Prevent left ventricular Outlet ObstructioN; MR, mitral regurgitation; 3D, threedimensional; LVOT, left ventricular outflow tract.

3D TEE imaging can be a useful adjunct to guiding the catheter through the prosthetic valve orifice. A large $3 \mathrm{D}$ sampling volume of the mitral valve prosthesis and LA is captured to allow for complete visualization of the catheter through the trans-septal puncture and its position in space above the mitral valve prosthesis.

With ViV TMVR, the aorto-mitral curtain is protected by the valve sewing ring. This allows for the simpler tipto-base LAMPOON method, bypassing the challenge of traversing the basal anterior mitral valve leaflet (14). In a retrospective cohort of 21 patients with either a prior mitral valve prosthesis or mitral ring annuloplasty who were at risk for LVOTO (defined as a neo-LVOT area $<2 \mathrm{~cm}^{2}$ ) and at prohibitive risk for redo mitral valve surgery, this method was shown to be both safe and effective. Of the 11 patients with a prior mitral valve prosthesis who underwent a tipto-base LAMPOON, none had LVOTO post-TMVR and none experienced any injury to the adjacent aortic root or aortic valve (15).

To form the flying $\mathrm{V}$ to lacerate the mitral valve prosthetic leaflet, a guide catheter in the LA and a guide catheter in the aorta are connected by a trans-septal guidewire. This guidewire is positioned at the tip of the prosthetic mitral valve leaflet. $3 \mathrm{D}$ TEE is often used to position the catheters to the appropriate central alignment of the leaflet. Multiplane TEE imaging can further refine the positioning of the catheter for increased chance of laceration success. Once in position, traction is applied on both catheters with laceration of the anterior leaflet from its tip to the base. The laceration is complete when the guidewire reaches the base of the mitral valve prosthesis (Figure 8).

Next, TEE is used to check for proper alignment of the laceration. An eccentric laceration may not be effective in preventing LVOTO. The surrounding structures to the mitral valve, particularly the aortic root, are investigated for any evidence of injury from the laceration attempt. The TMVR is then delivered into the surgical prosthesis as described in the previous section.

\section{Role of TEE for ViV in prior surgical tricuspid valve replacement (STVR)}

Prior to transcatheter tricuspid valve replacement (TTVR), pre-procedure MDCT is performed to assist in planning 

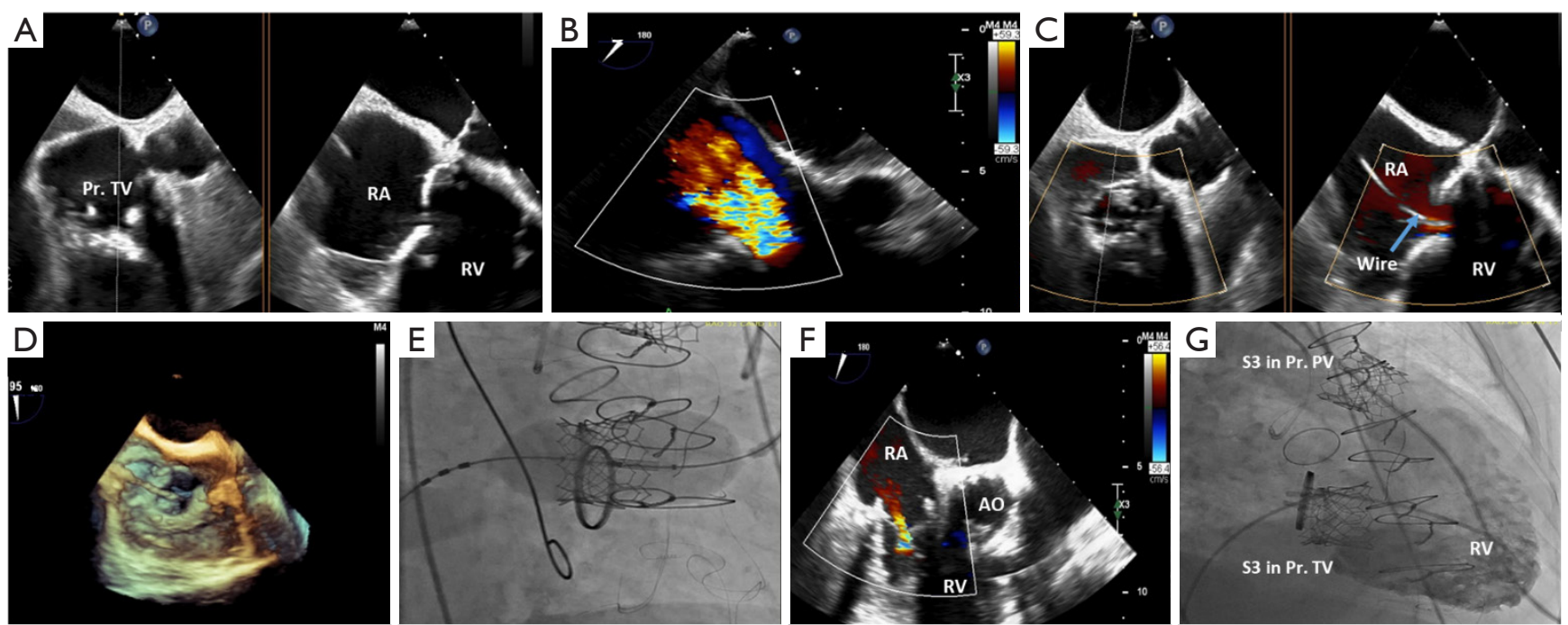

Figure 9 TEE and fluoroscopy guidance of transcatheter valve in a degenerated surgical tricuspid valve with severe TR. Severely degenerated surgical tricuspid valve prosthesis in the inflow/outflow view and 4 chamber views (A) with severe TR by color Doppler (B). A wire is seen crossing the tricuspid prosthesis $(\mathrm{C})$ with subsequent deployment of the transcatheter valve. 3D TEE (D) and fluoroscopic image (E) of the transcatheter valve being deployed in the prior prosthesis. Final assessment of the valve shows mild residual TR (F) and final position by fluoroscopy (G). RA, right atrium; RV, right ventricle; TEE, transesophageal echocardiography; TR, tricuspid regurgitation; 3D, three-dimensional.

with regard to valve sizing and potential complications. In most cases, ViV TTVR in a STVR can be performed using conscious sedation and local anesthesia with transthoracic or intracardiac echocardiographic guidance (19). Fluoroscopy provides the landmarks for $\mathrm{ViV}$ positioning and guidance during deployment. Echocardiography is used for assessment of the valve before and after valve deployment. TEE is often not necessary, and if used, may require that the patient be under general anesthesia. One scenario where TEE is recommended for ViV TTVR is when both valvular pathology and paravalvular leak are present. TEE should be used to determine the stability of the prosthesis as well as for planning of paravalvular leak closure (19).

\section{TEE guidance in ViV TTVR}

Tricuspid valve evaluation can be challenging due to the anterior position of the valve. Adequate visualization of the tricuspid valve prosthesis is further compounded in patients with a lipomatous interatrial septum, aortic or mitral prosthetic valve replacements, or a previous septal closure due to shadow artifact obscuring portions of the tricuspid valve. The tricuspid valve is evaluated in the standard views as described in Figure 1. In addition to these views, the modified bicaval view, which is typically located 10-20 degrees below the standard bicaval view, is occasionally the best window for assessment of the tricuspid valve. Any catheters or pacemaker leads also need to be noted at baseline.

TTVR generally uses the transfemoral approach. Modern day delivery systems are more directable and flexible, allowing them to overcome the acute angulation from the inferior vena cava to the tricuspid valve. The internal jugular vein may be used in tricuspid valves with an extremely horizontal access as this trajectory allows for a more coaxial position for valve deployment (19). After valve deployment, the new valve is assessed for any residual regurgitation, paravalvular leak, baseline transvalvular gradients, and pericardial effusion (Figure 9).

\section{Role of TEE for ViV in prior surgical pulmonic valve replacement (SPVR)}

As with all other $\mathrm{ViV}$ procedures, pre-procedural MDCT is used to determine transcatheter valve sizing and potential complications such as coronary compression during transcatheter pulmonary valve replacement. ViV procedures 

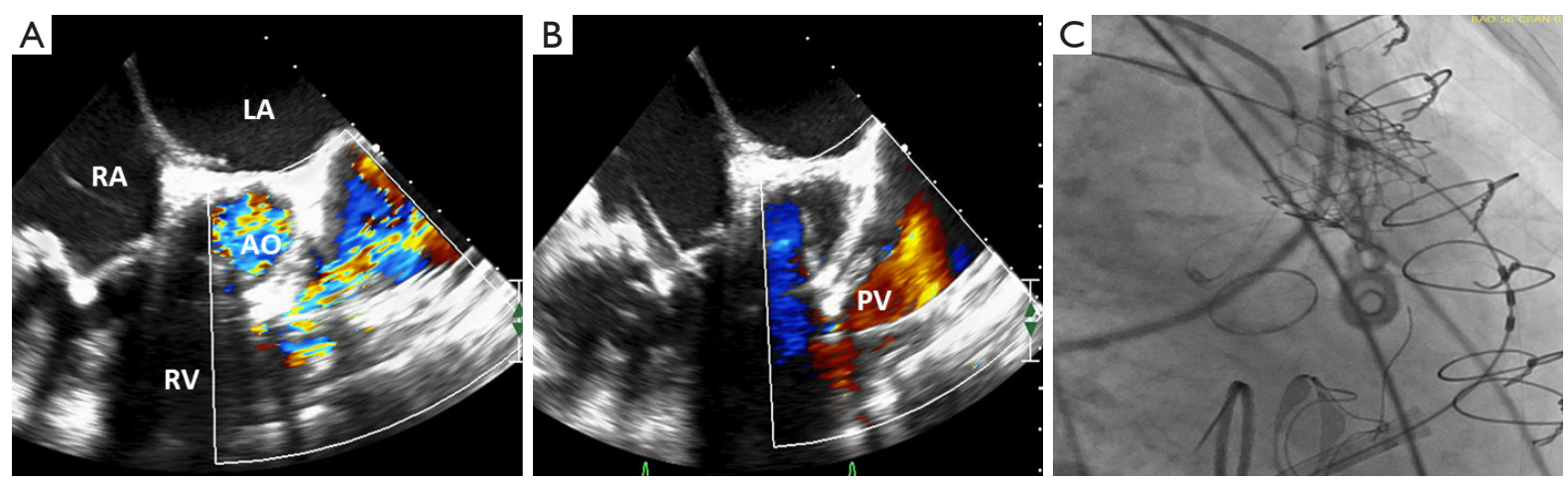

Figure 10 TEE and fluoroscopic images of transcatheter valve in prior SPVR. Baseline TEE image shows severe degeneration of the prosthetic pulmonic valve with turbulence by color Doppler (A). Post-deployment of the transcatheter valve, this turbulence has resolved (B) and the position of the pulmonic valve prosthesis can be seen on fluoroscopy (C). LA, left atrium; RA, right atrium; RV, right ventricle; TEE, transesophageal echocardiography; SPVR, surgical pulmonic valve replacement.

for SPVR are primarily guided by fluoroscopy. Due to its anterior and superior location, the pulmonary valve is often difficult to visualize well on TEE. TEE does not add significantly to the $\mathrm{ViV}$ positioning, deployment, or assessment of post-procedure complications beyond what fluoroscopy and TTE can identify. See Figure 10 for an example case.

\section{Conclusions}

TEE plays an important role in the peri- and intraprocedural guidance of $\mathrm{ViV}$ procedures. Upon valve deployment, TEE can assess the new prosthetic valve regarding its position within the prior surgical prosthesis, the transvalvular gradient, the degree of valvular regurgitation, and the degree of paravalvular regurgitation. Importantly, TEE aids not just in valve implantation, but also in assisting with transcatheter leaflet laceration to prevent catastrophic complications such as coronary obstruction or LVOTO.

\section{Acknowledgments}

We would like to thank Nirvi S. Bhargava for her careful editing of this manuscript.

Funding: None.

\section{Footnote}

Conflicts of Interest: The authors have no conflicts of interest to declare.
Open Access Statement: This is an Open Access article distributed in accordance with the Creative Commons Attribution-NonCommercial-NoDerivs 4.0 International License (CC BY-NC-ND 4.0), which permits the noncommercial replication and distribution of the article with the strict proviso that no changes or edits are made and the original work is properly cited (including links to both the formal publication through the relevant DOI and the license). See: https://creativecommons.org/licenses/by-nc$\mathrm{nd} / 4.0 /$.

\section{References}

1. Isaacs AJ, Shuhaiber J, Salemi A, et al. National trends in utilization and in-hospital outcomes of mechanical versus bioprosthetic aortic valve replacements. J Thorac Cardiovasc Surg 2015;149:1262-9.e3.

2. Kostyunin AE, Yuzhalin AE, Rezvova MA, et al. Degeneration of Bioprosthetic Heart Valves: Update 2020. J Am Heart Assoc 2020;9:e018506.

3. Mehaffey HJ, Hawkins RB, Schubert S, et al. Contemporary outcomes in reoperative mitral valve surgery. Heart 2018;104:652-6.

4. Maganti M, Rao V, Armstrong S, et al. Redo valvular surgery in elderly patients. Ann Thorac Surg 2009;87:521-5.

5. Khan JM, Greenbaum AB, Babaliaros VC, et al. The BASILICA Trial: Prospective Multicenter Investigation of Intentional Leaflet Laceration to Prevent TAVR Coronary Obstruction. JACC Cardiovasc Interv 2019;12:1240-52.

6. Ribeiro HB, Rodés-Cabau J, Blanke P, et al. Incidence, 
predictors, and clinical outcomes of coronary obstruction following transcatheter aortic valve replacement for degenerative bioprosthetic surgical valves: insights from the VIVID registry. Eur Heart J 2018;39:687-95.

7. Khan JM, Dvir D, Greenbaum AB, et al. Transcatheter Laceration of Aortic Leaflets to Prevent Coronary Obstruction During Transcatheter Aortic Valve Replacement: Concept to First-in-Human. JACC Cardiovasc Interv 2018;11:677-89.

8. Protsyk V, Meineri M, Kitamura M, et al. Echocardiographic Guidance of Intentional Leaflet Laceration prior to Transcatheter Aortic Valve Replacement: A Structured Approach to the Bioprosthetic or Native Aortic Scallop Intentional Laceration to Prevent Iatrogenic Coronary Artery Obstruction Procedure. J Am Soc Echocardiogr 2021;34:676-89.

9. Komatsu I, Leipsic J, Webb JG, et al. Coronary ostial eccentricity in severe aortic stenosis: Guidance for BASILICA transcatheter leaflet laceration. J Cardiovasc Comput Tomogr 2020;14:516-9.

10. Yoon SH, Bleiziffer S, Latib A, et al. Predictors of Left Ventricular Outflow Tract Obstruction After Transcatheter Mitral Valve Replacement. JACC Cardiovasc Interv 2019;12:182-93.

11. Hensey M, Brown RA, Lal S, et al. Transcatheter Mitral Valve Replacement: An Update on Current Techniques, Technologies, and Future Directions. JACC Cardiovasc Interv 2021;14:489-500.

12. Yoon SH, Whisenant BK, Bleiziffer S, et al. Outcomes of transcatheter mitral valve replacement for degenerated bioprostheses, failed annuloplasty rings, and mitral annular calcification. Eur Heart J 2019;40:441-51.

Cite this article as: Bhargava AA, Shekiladze N, Xie J, Kini A, Gleason PT, Lerakis S. Use of transesophageal echocardiography for transcatheter valve-in-valve implantation for patients with prior bioprosthetic surgical aortic, mitral, tricuspid, and pulmonic valves. Ann Cardiothorac Surg 2021;10(5):605-620. doi: 10.21037/acs-2021-tviv-27
13. Guerrero M, Vemulapalli S, Xiang Q, et al. Thirty-Day Outcomes of Transcatheter Mitral Valve Replacement for Degenerated Mitral Bioprostheses (Valve-in-Valve), Failed Surgical Rings (Valve-in-Ring), and Native Valve With Severe Mitral Annular Calcification (Valve-in-Mitral Annular Calcification) in the United States: Data From the Society of Thoracic Surgeons/American College of Cardiology/Transcatheter Valve Therapy Registry. Circ Cardiovasc Interv 2020;13:e008425.

14. Case BC, Lisko JC, Babaliaros VC, et al. LAMPOON techniques to prevent or manage left ventricular outflow tract obstruction in transcatheter mitral valve replacement. Ann Cardiothorac Surg 2021;10:172-9.

15. Lisko JC, Babaliaros VC, Khan JM, et al. Tip-to-Base LAMPOON for Transcatheter Mitral Valve Replacement With a Protected Mitral Annulus. JACC Cardiovasc Interv 2021;14:541-50.

16. Natarajan N, Patel P, Bartel T, et al. Peri-procedural imaging for transcatheter mitral valve replacement. Cardiovasc Diagn Ther 2016;6:144-59.

17. Elliott PM, Gimeno JR, Tomé MT, et al. Left ventricular outflow tract obstruction and sudden death risk in patients with hypertrophic cardiomyopathy. Eur Heart J 2006;27:1933-41.

18. Harloff MT, Chowdhury M, Hirji SA, et al. A step-bystep guide to transseptal valve-in-valve transcatheter mitral valve replacement. Ann Cardiothorac Surg 2021;10:113-21.

19. Sanon S, Cabalka AK, Babaliaros V, et al. Transcatheter Tricuspid Valve-in-Valve and Valve-in-Ring Implantation for Degenerated Surgical Prosthesis. JACC Cardiovasc Interv 2019;12:1403-12. 